\title{
Research on the Influence of Seeding Covered Peanut on the Germination of Peanut
}

\author{
Huafang Yu \\ Loudi Vocational and Technical College, Loudi Hunan, 417000, China
}

Key Words: Peanut, Seeding Covered Peanut, Agricultural Activities, Emergence Rate

\begin{abstract}
Most people tend to seed with shellless peanuts. This seeding method is favorable. First, people can pick good seeds first. Second, as long as the soil temperature adapts to the soil moisture content, the seeds will germinate fast and regularly. However, everything has two sides. In case of drought or high humidity, or other atrocious weather, the broadcasted shellless seeds are liable to be damaged, going moldy or rotting, which will directly affect the emergence rate and the seedling growth of peanut. In order to solve this problem, a method of seeding covered peanut is adopted. This method is favorable for protecting seeds. In this article, the influence of this method on the germination of peanut will be discussed in three parts: advantages of seeding covered peanut; influence of seeding covered peanut on the emergence rate, seedling uniformity degree, and seedling growth, including analysis of the test and conclusion of seeding covered peanut; points for attention in seeding covered peanut, with a hope to provide a useful guidance to the research of agricultural activities like seeding covered peanut.
\end{abstract}

\section{Introduction}

Peanut is a therophyte of papilionaceae arachis linn., It is also called "longevity nut" for it is rich in nutrition, nourishing, and favorable for prolonging life; and is called "vegetable meat" for its content of oil reaches up to $50 \%$. Besides eating, peanut also can be used in textile printing and papermaking, of certain industrial value; and for treating malnutrition and disorder of spleen and stomach, of certain medical value. As one of the major oil-bearing crops in China, peanut is widely planted in China, of which the growing scope starts from Fuyuan in Heilongjiang in the east, to Shache in Xinjiang in the west, to Yaxian County in Hainan to the south, and to Gannan in Heilongjiang in the north, covering a large area of China. In regard of the planting of peanut, weather and the condition of cropland are the key to the yield of peanut. For example, the spring in the north region of China is dry. If you want to seed after raining, the best sowing time is likely to be delayed, which will reduce the yield of peanut. Hence, how to increase of the yield of peanut becomes a focal point. With the traditional seeding method, namely seeding shellless peanut, some common problems often occur, such as seeds going moldy or decaying in case of atrocious weather, so that a high emergence rate cannot be guaranteed, and the growth of seedling also will be affected. A method of seeding covered peanut, which is helpful to overcome the aforesaid problems, becomes discussible here.

\section{Analysis of Advantages of Seeding Covered Peanut}

First, comparing with the traditional method of seeding shellless peanut, the obvious advantage of the method of seeding covered peanut apparently is that the shelling work becomes unnecessary, which reduces the quantity of work and saves the time of farmers.

Second, shellless peanut seeds are liable to be damaged in atrocious weather, while the peanut shell can protect the seeds, which is a protective layer. Specifically, peanut shell can protect the seeds from going moldy or decaying, and enhance the disease resistance by reducing the disease incidence at least by $20 \%$ to $30 \%$, so that the peanut seedlings can grow stably and healthily, favorable for yield increase. Practice shows that the rate of growth can be reach at least $10 \%$ to $15 \%$ by adopting the method of seeding covered peanut.

Third, peanut shell will provide a good ventilation condition for the seeds, which also can increase the emergence rate. Peanut seedlings will bear certain pressure when coming up, so that their roots 
can thrive downwards to obtain a strong vigor. However, it is not the case when the method of seeding shellless peanut is adopted. Generally, the emerging ability of shellless peanut seeds is lower than that of covered peanut. More worsely, the shellless peanut seeds will lose its germinating ability, because they will absorb the moisture in the air, and their respiratory action and the enzymatic activity will become more active, which will reduce the vigor of peanut seeds.

Fourth, peanut shell is rich in various mineral elements, such as phosphorus, nitrogen, and potassium, which will nourish the seeds before and during the emergence of seedlings, providing nutrition for the growth of the seedlings. Thus, it is not necessary to fertilize the seeds at the time of seeding.

\section{Influence of Seeding Covered Peanut on the Emergence Rate, Seedling Uniformity Degree, and Seedling Growth}

\section{Experiment of Seeding Covered Peanut}

The influence of seeding covered peanut on the emergence rate, seedling uniformity degree and seedling growth can be analyzed through experiment, as below:

Covered peanut seeds should be soaked for 1 to 2 days, there are two depths of seeding, namely $5 \mathrm{~cm}$ and $7 \mathrm{~cm}$, and there are two seeding modes, namely rolling and not rolling. On this basis, the experiment is designed to proceed with 8 groups.

The first group soak the seeds 1 day, sow seeds $5 \mathrm{~cm}$ deep, and roll the seeds; the second group soak the seeds 1 day, sow seeds $5 \mathrm{~cm}$ deep, and do not roll the seeds; the third soak the seeds 1 day, sow seeds $7 \mathrm{~cm}$ deep, and roll the seeds; the fourth group soak the seeds 1 day, sow seeds $7 \mathrm{~cm}$ deep, and do not roll the seeds.

The fifth group soak the seeds 2 days, sow seeds $5 \mathrm{~cm}$ deep, and roll the seeds; the sixth group soak the seeds 2 days, sow seeds $5 \mathrm{~cm}$ deep, and do not roll the seeds; the seventh soak the seeds 2 days, sow seeds $7 \mathrm{~cm}$ deep, and roll the seeds; the eighth group soak the seeds 2 days, sow seeds $7 \mathrm{~cm}$ deep, and do not roll the seeds.

Through experiment, it is found that under the same conditions of seed soaking and rolling, the seedlings of the seeds sowed $5 \mathrm{~cm}$ deep come up faster than that sowed $7 \mathrm{~cm}$ deep; under the same conditions of seeding depth and rolling, the seedlings of the seeds soaked 1 day come up faster than that soaked 2 days; and under the same conditions of seeding depth and seed soaking, the seedlings of the seeds rolled come up faster than that not rolled.

The experiment shows that the seedlings of the fifth group come up fastest, followed by the first group, and then the sixth group, the second group, the third group, the seventh group eighth group, and the fourth group.

Besides, the emergence rate, the seedling uniformity degree and the seedling growth of the seeds sowed $5 \mathrm{~cm}$ deep are better; under the same conditions of rolling and seeding depth, the emergence rate, the seedling uniformity degree and the seedling growth of the seeds soaked 2 days are better; and under the same conditions of seeding depth and seed soaking, the emergence rate, the seedling uniformity degree and the seedling growth of the seeds rolled are better.

\section{Analysis of Experiment Results of Seeding Covered Peanut}

To sum up, the emergence rate, the seedling uniformity degree and the seedling growth of the seeds of the fifth group are better than that of other groups. Thus, we can summarize more scientific and rational conditions for seeding covered peanut, under which the method of seeding covered peanut is superior to that on other conditions, as below:

Function of Bacteria Inhibition of Peanut Shell

The tannin substance contained in peanut shell is helpful to inhibit diseases, prevent the seeds from being damaged by bacteria, and reduce the damage of pests in soil to the seeds, guaranteeing the health of the seeds and of the seedlings. 


\section{Function of Seed Vigor Protection of Peanut Shell}

The peanut shell can prevent the decline of the vigor of the seeds. Seed vigor is an important criterion for seed certification, covering: (1) the speed of germinating; (2) the uniformity degree of seedlings; (3) the growth situation of the seedlings; (4) the resistance of the seedlings to inverse force; (5) the adaptive capacity of the seedlings to the external environment, etc. Therefore, if the method of seeding covered peanut is adopted, the seed vigor can be protected, and the speed of germinating, the uniformity degree of seedlings and the growth situation of the seedlings can be guaranteed, so that the seedlings are strong enough to resist diseases and adapt to the external environment.

Function of Protecting Seedling of Peanut Shell under External Atmosphere

Seeding covered peanut is favorable for reducing the influence of external gas environment on the germinating of seeds. Besides, the seeds can absorb sufficient moisture in the process of seed soaking, which is favorable for the survival of the seeds under atrocious weather like spring drought, and avoiding germination failure of seeds due to drought.

Function of Nutrient Conservation of Peanut Shell

Peanut is rich in nutrition, and directly soaking covered peanut can prevent the nutrition loss. Generally, the shellless peanut will turn red brown after being soaked, and becomes sticky. Under such conditions, the nutrition contained in peanut will exudate with water, causing nutrition loss. If the method of seeding covered peanut, the nutrition loss can be considerably reduced, because the peanut shell can prevent the nutrition from exudating, which can provide nutrition for the seeds during the period of geminating, boost the seedlings to thrive, and increase the planting and yield of peanut.

Fertilizer-saving

If excessive fertilizers are applied, the seedlings will be damaged, such as seedling burnt phenomenon. If the method of seeding covered peanut, it is unnecessary to apply fertilizer, because the tannin substance and fiber nutrients contained in peanut shell are enough for the growth of the seedlings, which can guarantee the emergence rate of peanut. In some rural areas where the base installations are poor, the resistance of the farmland soil and other external environment factors for peanut planting to disasters is low. For example, the soil fertility is low, and the soil contains a little organic matters. In such areas, the advantages of the method of seeding covered peanut will become more prominent.

\section{Points for Attention in Seeding Covered Peanut}

First, pick the early-maturing seeds, and dry them in the sun for about 2 or 3 days, so as to facilitate the metabolic activities of the seeds, enhance the osmotic pressure and the water absorbing capacity of the seeds, and increase the emergence rate. Separate the kernels of peanut in the process of drying. Squeeze the peanut shell, so that the kernels can contact air, and the water absorbing and ventilation of the kernels can be facilitated, favorable for the germinating. Generally, in comparison with the seeds not dried in the sun, the seedlings of the seeds dried in the sun can come up faster by 1 or 2 days, and the seedlings are neater and healthier.

Second, the sowing time of covered peanut should be earlier than that of shellless peanut by about 20 days. If the sowing time is delayed, the effect of yield increase cannot be achieved. Generally, if the method of seeding covered peanut is adopted, the seedlings will grow faster at the phase of germinating, and slow in the middle and later periods, both the seedlings and the stem are short, and the seedlings are growing compactly. Therefore, in order to let the seedlings develop better, the spaces among each seedling should be increased by $10 \%$ to $15 \%$, so as to realize yield increase.

Third, soak the prepared peanut seeds in warm water at $35^{\circ} \mathrm{C}$ for 6 to 8 hours, so that the peanut shell can absorb enough water, and become soft; then drain the soaked seeds, and sow the seeds, so as to increase the emergence rate.

Fourth, sows the seeds shallowly at the depth of $3 \mathrm{~cm}$ to $5 \mathrm{~cm}$, because the covered peanut germinates slower than the shellless peanut does. It is feasible to sow two to three seeds once. Cover the seeds with soil, and properly roll them, so that the seedlings will not come up too slow, or the 
peanut shell will not come out of soil. If any seedling comes up bearing the shell, remove the shell promptly.

Fifth, take good care of the seedlings, by regularly or irregularly observing the growth condition of the seedlings, taking measures for facilitating germination to avoid excessive weak seedlings or dead seedlings, and taking measures for preventing plant diseases and insect pests.

\section{Conclusion}

Peanut cultivation has a long history in China. There are many problems in the traditional method of seeding shellless peanut. In comparison, the method of seeding covered peanut is superior, and is a new technology of peanut cultivation in resisting drought, saving water and helping yield increase. The peanut shell, as a protective layer, can protect the seeds from damage by the external severe environment, prevent the seed vigor from declining, guarantee a high emergence rate, and realize yield increase. Therefore, picking early-maturing seeds, and pre-processing of seeds are the necessary preparatory works. Besides, sowing the seeds slightly early and shallowly with a depth of $3 \mathrm{~cm}$ to $5 \mathrm{~cm}$, taking good care of the seedlings, observing the growth condition of the seedlings, taking measures for preventing plant diseases and insect pests are necessary to boost the seedlings to growth healthily, and realize yield increase. To sum up, the method of seeding covered peanut has become a superior technology, worth popularizing.

\section{Acknowledgement}

Hunan provincial science and technology program project,Project No.: 2012NK3048, 2014 Loudi municipal science and technology program project, Project Name: Research on the Technology of Seeding Covered Peanut of Small Seeds in Xinhua County .

\section{References}

[1] ZHANG Zhimeng, CI Dunwei, DING Hong, QIN Feifei, YAGN Jishun, LI Shangxia, SONG Wenwu, DAI Liangxiang. Influence of Peanut Seed Size and Shape on Germination and Seedling Growth. Journal of Peanut Science, 2014, 01: 16-23.

[2] LYU Jianwei, MA Tianjin, HU Tinghui, CHEN Feng, LI Zhengqiang, JIAO Aixia. Problems of Peanut Germinating and Solutions thereto. Journal of Agricultural Science and Technology, 2015, 12: 231-233.

[3] CHANG Mei, ZHENG Yiqiang. Influence of Seeding Covered Peanut on Germination.Anhui Agricultural Sciences, 2013, 02: 505-506.

[4] YU Shuze. Advantages of Seeding Covered Peanut. New Rural Technology, 2013, 04: 5-7.

[5] WEI De. Seeding Covered Peanut—Favorable for Drought Resisting and Yield Increase. Famers Consultant, 2002, 05: 15-16. 